\title{
REVIEW
}

\section{Neurogenic pulmonary edema}

\author{
Danielle L Davison, Megan Terek and Lakhmir S Chawla* \\ This article is one of eleven reviews selected from the Annual Update in Intensive Care and Emergency Medicine 2012 (Springer Verlag, \\ DOI: 10.1007/978-3-642-25716-2) and co-published as a series in Critical Care. Other articles in the series can be found online at \\ http://ccforum.com/series/annualupdate2012. Further information about the Annual Update in Intensive Care and Emergency Medicine is \\ available from http://www.springer.com/series/8901.
}

\section{Introduction}

Neurogenic pulmonary edema (NPE) is a clinical syndrome characterized by the acute onset of pulmonary edema following a significant central nervous system (CNS) insult. The etiology is thought to be a surge of catecholamines that results in cardiopulmonary dysfunction. A myriad of CNS events, including spinal cord injury, subarachnoid hemorrhage (SAH), traumatic brain injury (TBI), intracranial hemorrhage, status epilepticus, meningitis, and subdural hemorrhage, have been associated with this syndrome [1-5]. Although NPE was identified over 100 years ago, it is still underappreciated in the clinical arena. Its sporadic and relatively unpredictable nature and a lack of etiologic-specific diagnostic markers and treatment modalities may in part be responsible for its poor recognition at the bedside. In this manuscript, we will review the anatomical origin of NPE, outline the various possible pathophysiologic mechanisms responsible for its development, and propose a clinical framework for the classification of NPE.

\section{Historical background}

The syndrome of NPE has been recognized for over a century. In 1903, Harvey Williams Cushing, described the connection between CNS injury and hemodynamic dysfunction [6]; and, in 1908, W. T. Shanahan reported 11 cases of acute pulmonary edema as a complication of epileptic seizures [7]. Francois Moutier described the sudden onset of pulmonary edema among soldiers shot in the head in World War I [8]. Similar reports exist of observed alveolar edema and hemorrhage in the lungs of 17 soldiers dying after isolated bullet head wounds in the Vietnam War [1].

*Correspondence: minkchawla@gmail.com

Department of Critical Care Medicine and Anesthesiology, George Washington University Medical Center, 900 23rd Street NW, Room G-105, Washington, DC 20037, USA

\section{Epidemiology}

Because much of the clinical information on NPE has been derived from case reports and autopsy series, the true incidence of NPE is unknown and is likely underreported. Any acute CNS insult, including spinal cord trauma, can result in pulmonary edema. In patients with SAH, reports of NPE incidence range from $2 \%$ to $42.9 \%$ $[3,9,10]$. Clinically, the likelihood of developing NPE following SAH correlates with increasing age, delay to surgery, vertebral artery origin, and the severity of clinical and radiographic presentation (e.g., Hunt-Hess and Fischer grades) [10,11]. Patients with SAH who develop NPE have a higher mortality rate, nearing $10 \%$ [3]. In patients with TBI, the incidence of NPE has been estimated to be up to $20 \%$ [12]. Rogers et al. examined a large autopsy and inpatient database on patients with acute head injury in an effort to better characterize NPE in this patient population. The authors found that the incidence of NPE in patients with TBI who died at the scene was $32 \%$. Among the TBI patients who died within 96 hours, the incidence of NPE rose to 50\% [2]. There was a direct correlation between decreasing cerebral perfusion pressure (CPP) and reduced $\mathrm{PaO}_{2} / \mathrm{FiO}_{2}$ ratios in the TBI patients [2]. NPE in patients who suffer seizures is rare; however, up to 80 to $100 \%$ of epileptics who die unexpectedly of seizures are also found to have NPE [13]. In other series, close to one-third of patients with status epilepticus also developed NPE [14]. Other conditions, including aqueductal glioma, multiple sclerosis, medication overdose, arteriovenous malformations, meningitis/ encephalitis and spinal cord infarction, have been reported and linked to the formation of NPE [5,15-17].

\section{Pathophysiology}

The pathophysiology linking the neurologic, cardiac, and pulmonary conditions in NPE has been subject to debate and controversy since the recognition of NPE as a clinical entity. A common thread among all case descriptions of 
NPE is the severity and acuity of the precipitating CNS event. Neurologic conditions that cause abrupt, rapid, and extreme elevation in intracranial pressure (ICP) appear to be at greatest risk of being associated with NPE $[18,19]$. Elevated ICP levels correlate with increased levels of extravascular lung water (EVLW) and NPE $[2,20]$. The abrupt increase in ICP leading to neuronal compression, ischemia or damage is believed to give rise to an intense activation of the sympathetic nervous system and the release of catecholamines [2,21]. This fundamental role of catecholamines is supported by the fact that the blockade of sympathetic activity in animal models via intrathecal lidocaine, phentolamine infusion, or pretreatment with phenoxybenzamine mitigates the pathologic neuro-pulmonary process [22,23] (Table 1 ). In addition to pharmacologic intervention, anatomical interruption of the nervous system pathway (e.g., spinal cord transection) has also been shown to protect against the formation of NPE (Table 1). In one animal model, NPE was prevented by removal of one lung followed by reimplantation. This was in contrast to the pulmonary edema that developed in the innervated intrinsic lung [24]. In a human example, NPE has been reported in soldiers who died suddenly after gunshot wounds to the head. The soldiers with concomitant cervical spinal cord injury (and presumably severed neuronal connection) did not have evidence of pulmonary edema on post-mortem exam [1]. Pulmonary edema has also been reported in patients with pheochromocytoma, presumably from catecholamine surge [25].

\section{Anatomical origin of NPE}

Although the exact source of sympathetic outflow has not been identified, certain centers in the brain have been implicated. These 'NPE trigger zones' include the hypothalamus and the medulla, specifically area A1, A5, nuclei of solitary tract and the area postrema [5]. Area A1 is located in the ventrolateral aspect of the medulla and is composed of catecholamine neurons which project into the hypothalamus [5]. The neurons from area A5, located in the upper portion of the medulla, project into the preganglionic centers for spinal cord sympathetic outflow [5]. Injury to Area A1 or disruption of the efferent pathway between A5 and the cervical cord has been shown to result in the formation of pulmonary edema [26]. Stimulation of area A5 also causes increases in systemic blood pressure [27]. The nuclei of solitary tract and the area postrema of the medulla have also been linked to the formation of NPE. These areas are related to respiratory regulation and receive input from the carotid sinus. In animal models, bilateral irritation of the nuclei solitary tract causes severe hypertension and NPE [23]. Unilateral stimulation of the area postrema also results in profound hemodynamic changes, including increased cardiac output, peripheral vascular resistance, and hypertension [5]. Finally, NPE was shown to develop after lesions were induced in the hypothalamus of laboratory animals [28]. In a case series of 22 patients suffering from NPE, 11 of the patients had significant radiographic abnormalities in the hypothalamus. The presence of hypothalamic lesions among these NPE patients conferred a worse prognosis [29].

\section{Pathogenesis}

It is the prevailing view that the autonomic response to elevated ICP plays an important role in the pathogenesis of NPE. However, what occurs mechanistically at the level of the pulmonary vascular endothelium remains enigmatic and theoretical. Several clinicopathologic paradigms have been proposed to explain the clinical syndrome of NPE: 1) Neuro-cardiac; 2) Neuro-hemodynamic; 3) "blast theory"; and 4) pulmonary venule adrenergic hypersensitivity.

\section{Neuro-cardiac NPE}

Whereas NPE has traditionally been described as a 'noncardiogenic' form of pulmonary edema, there is evidence that, in at least a subset of patients, neurologic insult leads to direct myocardial injury and the development of pulmonary edema. Takotsubo's cardiomyopathy is a reversible condition characterized by depressed cardiac contractility following a neurologically 'stressful' event. The transiently diminished lusitropy, diastolic dysfunction, and global hypokinesis of the Takotsubo heart can render these patients susceptible to cardiogenic pulmonary edema [30]. Connor was one of the first investigators to describe the myocytolysis and contraction-band necrosis on myocardial biopsies of neurosurgical patients with pulmonary edema [31]. Since this original report, several cases of cardiac injury associated with pulmonary edema following a CNS event have been described. In a retrospective analysis, patients with no previous cardiac history developed acute onset of pulmonary edema in association with a SAH. The patients all demonstrated segmental wall motion abnormalities on echocardiogram, mildly elevated cardiac enzymes, electrocardiogram (EKG) abnormalities, and elevated pulmonary artery occlusion pressures (PAOPs). These patients were noted to have focal myocardial necrosis, yet had no evidence of infarction and had normal coronary arteries [32]. Similar descriptions of reversible cardiac dysfunction have been reported among patients with TBI and NPE [30].

As with all forms of NPE, massive sympathetic discharge following CNS insult is thought to be the precipitating factor. More specifically, in this subset of patients with 'neuro-cardiac' NPE, it is catecholamines that induce direct myocyte injury. This is supported by the fact that the wall motion abnormalities seen on 
Table 1. Animal studies assessing possible therapeutic interventions for neurogenic pulmonary edema (NPE)

\begin{tabular}{|c|c|c|}
\hline Intervention & Animal model & Study design/results \\
\hline \multicolumn{3}{|l|}{ Alpha Blockade } \\
\hline Phentolamine (1 mg/kg) & Rats & Prevented pulmonary edema after induced injury to anterior hypothalamus [23] \\
\hline Phenoxybenzamine (3 mg/kg) & Dogs & $\begin{array}{l}\text { Prevented pulmonary artery and systemic pressure increase after CSF pressure was increased } \\
\text { from } 100 \text { to } 200 \mathrm{mmHg}[22]\end{array}$ \\
\hline Phenoxybenzamine (1.5 mg/kg) & Dogs & $\begin{array}{l}\text { Prevented increases in pulmonary perfusion pressure and PVR and associated increases in } \\
\text { lung water, Qs/Qt, VD, and hypoxemia induced by ICP elevation [50] }\end{array}$ \\
\hline Phentolamine (2 mg/kg) & Sheep & Prevented the expected increase in permeability and lymph flow after CNS insult [38] \\
\hline \multicolumn{3}{|l|}{ Beta Blockade } \\
\hline Propranolol (0.5 mg/kg) & Dogs & $\begin{array}{l}\text { Pulmonary artery and systemic pressure unchanged with use of beta blocker after CSF } \\
\text { pressure was increased from } 100 \text { to } 200 \mathrm{mmHg} \text { [22] }\end{array}$ \\
\hline Propranolol (1.5 mg/kg) & Dogs & $\begin{array}{l}\text { Pretreatment with beta blocker attenuated the increase in PVR during elevation in ICP but did } \\
\text { not prevent increases in lung water, Qs/Qt, VD, and hypoxemia [50] }\end{array}$ \\
\hline \multicolumn{3}{|l|}{ Sympathetic Outflow Denervation } \\
\hline Bilateral thoracic sympathectomy & Dogs \& Rabbits & $\begin{array}{l}\text { Sympathectomy prior to induced CNS insult did not prevent pulmonary pressure } \\
\text { elevation [35] }\end{array}$ \\
\hline Spinal cord transection & Monkeys & NPE prevented by sympathetic denervation [18] \\
\hline \multicolumn{3}{|l|}{ Cholinergic Influence } \\
\hline Vagotomy & Dogs \& Rabbits & Vagotomy did not prevent increases in pulmonary vascular pressures [35] \\
\hline Vagotomy & Monkeys & Vagotomy did not prevent NPE [18] \\
\hline \multicolumn{3}{|l|}{ Other } \\
\hline Methylprednisone (40 mg/kg) & Rats & Prevented aconitine induced NPE and systemic HTN [51] \\
\hline Hypovolemia & Rats & Lowering pulmonary blood volume by phlebotomy prevented aconitine induced NPE [51] \\
\hline Naloxone & Sheep & $\begin{array}{l}\text { Induced NPE could be prevented by opiate antagonism, suggesting a role for } \\
\text { endorphins [45] }\end{array}$ \\
\hline
\end{tabular}

CNS, central nervous system; CSF, cerebrospinal fluid; PVR, pulmonary vascular resistance; Qs/Qt, pulmonary shunt; VD, dead space.

echocardiogram in patients with neurogenic stunned myocardium follow a pattern of sympathetic nerve innervation [33]. Similarly, myocardial lesions have been shown in patients with pheochromocytoma, supporting the role of catecholamine surge in the pathogenesis of stunned myocardium [34].

\section{Neuro-hemodynamic NPE}

Unlike the direct toxic effects to the myocardium as detailed above, the 'neuro-hemodynamic' theory posits that ventricular compliance is indirectly altered by the abrupt increases in systemic and pulmonary pressures following CNS injury. In the original studies by Sarnoff and Sarnoff, substantial increases in aortic and pulmonary pressures were observed following the injection of thrombin into the intracisterna magna of dogs and rabbits [35]. The authors noted that following the sympathetic surge, the left ventricle had reached its workfailure threshold and failed to effectively pump against the systemic pressures. A translocation of blood flow from the highly resistant systemic circulation to the low resistance pulmonary circuit subsequently ensued, leading to a hydrostatic form of pulmonary edema. The increased sizes of the left atrium and pulmonary veins in the animals were well documented in this study, and the authors subsequently coined the term "neuro-hemodynamic pulmonary edema" [35]. Several other animal models have documented large elevations in left atrial, systemic and pulmonary pressures associated with NPE $[18,22,36]$. One study induced graded levels of ICP in chimpanzees. All of the animals developed systemic hypertension, but only those with a marked increase in left atrial pressure and a decrease in cardiac output developed pulmonary edema [18].

\section{Blast theory}

The neuro-cardiac and neuro-hemodynamic theories outlined above both suggest that alterations in hydrostatic and Starling forces are central to the formation of pulmonary edema following CNS injury. Although hydrostatic pressures may play a role in the pathogenesis, this mechanism alone cannot explain the presence of red blood cells (RBCs) and protein observed in the alveolar fluid in many NPE subjects [37,38]. The exudative properties of the pulmonary fluid imply that alterations in vascular permeability play a role in the pathogenesis of NPE. In order to explain the presence of both hydrostatic factors and vascular leak, Theodore and Robin introduced 
the "blast theory" of NPE [39]. Similar to the neurohemodynamic model, the "blast theory" posits that the severe abrupt increases in systemic and pulmonary pressures following the catecholamine surge result in a net shift of blood volume from the systemic circulation to the low resistance pulmonary circulation. This increase in pulmonary venous pressure leads to the development of transudative pulmonary edema. The "blast theory" further posits that the acute rise in capillary pressure induces a degree of barotrauma capable of damaging the capillary-alveolar membrane. The structural damage to the pulmonary endothelium ultimately leads to vascular leak and persistent protein-rich pulmonary edema [39]. The pulmonary edema according to the "blast theory" is thus the result of two mechanisms which act synergistically: A high-pressure hydrostatic influence and pulmonary endothelial injury. Several pre-clinical models support this mechanism $[40,41]$. Maron showed that barotrauma and vascular permeability occurred when pulmonary pressures exceeded 70 torr following CNS injury in dogs [40]. In another study, EVLW was observed when pulmonary artery pressures reached 25 torr or greater in rabbits [41]. The authors concluded that some degree of pulmonary hypertension is required for the development of pulmonary edema, and that the degree of permeability is "pressure dependent" [41].

Theodore and Robin in the "blast theory" acknowledged that it is rare to document elevated systemic and pulmonary pressures in human cases of NPE. According to their theory, this can be explained by the fact that the sympathetic surge and subsequent hemodynamic instability occurs at the time of the inciting event when hemodynamic monitoring is rare [39]. During the later stages of NPE, systemic and pulmonary pressures can return to normal, whereas the endothelial injury and vascular leak may persist [39]. A few case reports have been able to document this sequence of events in human subjects, lending credence to the "blast theory". One case study described a patient who had hemodynamic monitoring at the time of a seizure that led to NPE. Within minutes of the seizure, marked increases in systemic, pulmonary and pulmonary artery occlusion pressures were recorded. The hemodynamics quickly normalized and two hours later, pulmonary edema developed, which was determined to be high in protein content [37]. In another case report of a patient with an intracranial hemorrhage, extreme increases in systemic and mean pulmonary pressures $(410 / 200 \mathrm{mmHg}$ and $48 \mathrm{mmHg}$, respectively) lasted 4 minutes. This was followed by a dramatic decrease in the patient's oxygen levels. The patient's pulmonary edema did not clear on radiograph for 72 hours following the last episode of transient systemic and pulmonary hypertension. The authors concluded that persistent vascular leak was the basis for these findings [42].

\section{Pulmonary venule adrenergic hypersensitivity}

Many reports of NPE fail to consistently demonstrate the hypertensive surges and changes in left atrial pressures as described in the theories above. This suggests that systemic hypertension and its effect on cardiac contractility may not always contribute to the development of NPE. An alternative hypothesis is that the massive sympathetic discharge following CNS injury directly affects the pulmonary vascular bed, and that the edema develops regardless of any systemic changes. We refer to this as the 'pulmonary venule adrenergic hypersensitivity' theory. This concept of neurally induced changes to endothelial integrity is made plausible by the fact that pulmonary vascular beds contain $\alpha$ - and $\beta$-adrenergic receptors [43]. In a well designed study by McClellan et al. [44], CNS injury and elevated ICP was induced in dogs by cisternal saline infusion. Autonomic activation following the CNS insult was evidenced by an increase in systemic and pulmonary vascular pressures. The pulmonary edema developed in the dogs and was proven to be exudative in content. When the same degree of pulmonary hypertension and increased left atrial pressure was induced with a left atrial balloon in the control group, pulmonary edema did not develop. The authors concluded that neurologic insult resulted in acute lung injury (ALI), which could not be explained by hemodynamic changes, but rather by direct neurological influences on the pulmonary endothelium [44]. In other studies of intracranial lesions induced in sheep, pulmonary edema developed despite normal or only mildly increased left atrial and systemic pressures $[38,45]$. In one of these studies, $\alpha$-adrenergic blockade prevented the formation of pulmonary edema with little systemic effect, further supporting the role of direct adrenergic influence [38]. In human examples, continuous cardiac monitoring during the development of NPE in patients with SAH and brain tumor resection failed to demonstrate preceding hemodynamic changes [46-48]. These findings suggest that isolated pulmonary venoconstriction or endothelial disruption following CNS injury may be responsible for the formation of pulmonary edema $[47,48]$.

\section{Clinical characteristics}

Two distinct clinical forms of NPE have been described. The early form of NPE is most common and is characterized by the development of symptoms within minutes to hours following neurologic injury. In contrast, the delayed form develops 12 to 24 hours after the CNS insult [5]. The abrupt nature of respiratory distress is an impressive feature of NPE. Typically, the patient becomes acutely dyspneic, tachypneic, and hypoxic within minutes. Pink, frothy sputum is commonly seen and bilateral crackles and rales are appreciated on auscultation. Sympathetic hyperactivity is common and the patient 
may be febrile, tachycardic, and hypertensive, and leukocytosis may occur. Chest radiograph will reveal bilateral hyperdense infiltrates consistent with acute respiratory distress syndrome (ARDS) [5]. Symptoms often spontaneously resolve within 24 to 48 hours; however, in patients with ongoing brain injury and elevated ICP, the NPE often persists.

\section{Differential diagnosis}

Because alternative conditions are common, NPE is a difficult diagnosis to establish. The diagnosis of 'pure' NPE is a diagnosis of exclusion and, by traditional definition, requires documentation of non-cardiogenic pulmonary edema in the setting of neurological injury. Aggressive fluid hydration is frequently administered to neurologically injured patients. Large volume resuscitation is especially common in SAH patients suspected of having vasospasm, thus rendering these patients at risk for volume overload and pulmonary edema [9]. Aspiration pneumonia is also common among CNS injured patients and must be excluded. Aspiration pneumonia differs from NPE by the presence of clinical clues (vomiting, gastric contents in the oropharynx, witnessed aspiration) and the distribution of alveolar disease in dependent portions of the lungs. In contrast, NPE is characterized by a frothy, often blood-tinged sputum and more centrally distributed alveolar disease on radiograph $[5]$.

\section{Previous treatment in humans}

Although numerous case reports have described the various precipitating CNS insults and clinical scenarios associated with NPE, few studies have identified specific treatment modalities for this condition. The management of NPE to date has largely focused on treating the underlying neurologic condition in order to quell the sympathetic discharge responsible for causing the lung injury. Treatment efforts to reduce ICP, including decompression and clot evacuation, osmotic diuretics, anti-epileptics, tumor resection, and steroids have all been associated with improvements in oxygenation $[3,16,28]$. Pharmacological intervention, specifically anti$\alpha$-adrenergic agents, which potentially interrupt the vicious cycle of hemodynamic instability and subsequent respiratory failure, has shown promise in animal models. However, there are few reports documenting its use in humans. In one case report, a patient with TBI developed sudden onset of bilateral infiltrates and hypoxia in the setting of elevated blood pressures, sinus tachycardia and normal central venous pressure (CVP). This patient was successfully treated with the $\alpha$-blocking agent, chlorpromazine, as evidenced by rapid improvement in oxygenation and hemodynamics; catecholamine levels were not measured in this report [49].

\section{Proposed clinical framework, diagnostic criteria, and management of NPE}

NPE is an exotic form of pulmonary edema and can be considered a form of ARDS per the consensus definition. While all cases of NPE follow a CNS event and likely originate from sympathetic activation, downstream effects on the cardiopulmonary system vary. Some patients may have direct myocardial injury resulting in left ventricular failure and pulmonary edema. Others develop pulmonary edema from a non-cardiogenic mechanism as described in the pulmonary venule hypersensitivity models. Differentiating between 'cardiogenic involvement' and 'non-cardiogenic' mechanisms is essential in the clinical realm, as there are clear therapeutic implications. In order for this clinical entity to be effectively studied and treated, a definition that captures a subset of patients with NPE who may benefit from sympathetic interference would be helpful. We, therefore, propose the following diagnostic criteria for this subset of NPE: 1) Bilateral infiltrates; 2) $\mathrm{PaO}_{2} / \mathrm{FiO}_{2}$ ratio < 200; 3) no evidence of left atrial hypertension; 4) presence of CNS injury (severe enough to have caused significantly increased ICP); 5) absence of other common causes of acute respiratory distress or ARDS (e.g., aspiration, massive blood transfusion, sepsis). For those patients who meet the above NPE criteria, measurement of serum catecholamines may be helpful. In those patients in whom blood pressure permits, a trial of an $\alpha$-adrenergic blocking agent, such as phentolamine, can be considered.

\section{Conclusion}

Despite decades of scientific experiments and case descriptions, the diagnosis and management of NPE remains controversial and challenging. Although this syndrome has been described for over a millennium, it remains underdiagnosed and underappreciated. The exact pathophysiology of NPE is still debated and the wide variety of clinical situations in which it occurs can obfuscate diagnosis. The sudden development of hypoxemic respiratory failure following a catastrophic CNS event, which cannot be attributed to other causes of ARDS, is the only universally agreed upon characteristic of NPE. A common denominator in all cases of NPE is likely a surge in endogenous serum catecholamines that may result in changes in cardiopulmonary hemodynamics and Starling forces. It appears that the specific clinical manifestations of this surge may vary depending on the individual circumstance. In some patients, cardiac dysfunction may predominate; in others, capillary leak is the primary manifestation. These patterns have obvious implications for the diagnosis and treatment of individual cases, including cardiac evaluation, fluid management, and choice of inotropic or vasoactive substances such as $\alpha$ adrenergic blockade. 


\section{Abbreviations}

ALI, acute lung injury; ARDS, acute respiratory distress syndrome; CNS, central nervous system; CPP, cerebral perfusion pressure; CSF, cerebrospinal fluid; CVP, central venous pressure; EKG, electrocardiogram; EVLW, extravascular lung water; ICP, intracranial pressure; NPE, neurogenic pulmonary edema; PAOPS, pulmonary artery occlusion pressures; PVR, pulmonary vascular resistance; QS/ Qt, pulmonary shunt; RBCs, red blood cells; SAH, subarachnoid hemorrhage; $T B I$, traumatic brain injury; VD, dead space.

\section{Competing interests}

The authors declare that there are no competing interests.

Published: 20 March 2012

\section{References}

1. Simmons RL, Heisterkamp CA 3rd, Collins JA, Genslar S, Martin AM Jr: Respiratory insufficiency in combat casualties. 3. Arterial hypoxemia after wounding. Ann Surg 1969, 170:45-52.

2. Rogers FB, Shackford SR, Trevisani GT, Davis JW, Mackersie RC, Hoyt DB: Neurogenic pulmonary edema in fatal and nonfatal head injuries. J Trauma 1995, 39:860-866.

3. Fontes RB, Aguiar PH, Zanetti MV, Andrade F, Mandel M, Teixeira MJ: Acute neurogenic pulmonary edema: case reports and literature review. J Neurosurg Anesthesiol 2003, 15:144-150.

4. Kaufman HH, Timberlake G, Voelker J, Pait TG: Medical complications of head injury. Med Clin North Am 1993, 77:43-60.

5. Colice GL: Neurogenic pulmonary edema. Clin Chest Med 1985, 6:473-489.

6. Cushing $\mathrm{H}: \mathrm{I}$. On the avoidance of shock in major amputations by cocainization of large nerve-trunks preliminary to their division. With observations on blood-pressure changes in surgical cases. Ann Surg 1902, 36:321-345

7. Shanahan W: Acute pulmonary edema as a complication of epileptic seizures. NY Med J 1908, 37:54-56.

8. Moutier F: Hypertension et mort par oedeme pulmo aigu chez les blesses cranio-encephaliques. Presse Méd 1918, 26:108-109.

9. Friedman JA, Pichelmann MA, Piepgras DG, et al:: Pulmonary complications of aneurysmal subarachnoid hemorrhage. Neurosurgery 2003, 52:1025-1031.

10. Solenski NJ, Haley EC Jr, Kassell NF, et al:: Medical complications of aneurysmal subarachnoid hemorrhage: a report of the multicenter, cooperative aneurysm study. Participants of the Multicenter Cooperative Aneurysm Study. Crit Care Med 1995, 23:1007-1017.

11. Ochiai $H$, Yamakawa $Y$, Kubota E: Deformation of the ventrolateral medulla oblongata by subarachnoid hemorrhage from ruptured vertebral artery aneurysms causes neurogenic pulmonary edema. Neurol Med Chir (Tokyo) 2001, 41:529-534.

12. Bratton SL, Davis RL: Acute lung injury in isolated traumatic brain injury. Neurosurgery 1997, 40:707-712.

13. Wayne SL, O'Donovan CA, McCall WV, Link K: Postictal neurogenic pulmonary edema: experience from an ECT model. Convuls Ther 1997 13:181-184

14. Simon RP: Neurogenic pulmonary edema. Neurol Clin 1993, 11:309-323.

15. Gentiloni N, Schiavino D, Della Corte F, Ricci E, Colosimo C: Neurogenic pulmonary edema: a presenting symptom in multiple sclerosis. Ital J Neurol Sci 1992, 13:435-438.

16. Phanthumchinda K, Khaoroptham S, Kongratananan N, Rasmeechan S: Neurogenic pulmonary edema associated with spinal cord infarction from arteriovenous malformation. J Med Assoc Thai 1988, 71:150-153.

17. Wagle VG, Hall A, VoytekT, Silberstein H, Uphoff DF: Aqueductal (pencil) glioma presenting as neurogenic pulmonary edema: a case report. Surg Neurol 1990, 34:435-438.

18. Ducker TB, Simmons RL: Increased intracranial pressure and pulmonary edema. 2. The hemodynamic response of dogs and monkeys to increased intracranial pressure. J Neurosurg 1968, 28:118-123.

19. Kosnik EJ, Paul SE, Rossel CW, Sayers MP: Central neurogenic pulmonary edema: with a review of its pathogenesis and treatment. Childs Brain 1977, 3:37-47.

20. Gupta YK, Chugh A, Kacker V, Mehta VS, Tandon PN: Development of neurogenic pulmonary edema at different grades of intracranial pressure in cats. Indian J Physiol Pharmacol 1998, 42:71-80.

21. Demling R, Riessen R: Pulmonary dysfunction after cerebral injury. Crit Care
Med 1990, 18:768-774.

22. Brashear RE, Ross JC: Hemodynamic effects of elevated cerebrospinal fluid pressure: alterations with adrenergic blockade. J Clin Invest 1970, 49:1324-1333

23. Nathan $M A$, Reis $D$ J: Fulminating arterial hypertension with pulmonary edema from release of adrenomedullary catecholamines after lesions of the anterior hypothalamus in the rat. Circ Res 1975, 37:226-235.

24. Sugg WL, Craver WD, Webb WR, Ecker RR: Pressure changes in the dog lung secondary to hemorrhagic shock: protective effect of pulmonary reimplantation. Ann Surg 1969, 169:592-598.

25. de Leeuw PW, Waltman FL, Birkenhager WH: Noncardiogenic pulmonary edema as the sole manifestation of pheochromocytoma. Hypertension 1986, 8:810-812

26. Blessing WW, West MJ, Chalmers J: Hypertension, bradycardia, and pulmonary edema in the conscious rabbit after brainstem lesions coinciding with the A1 group of catecholamine neurons. Circ Res 1981 49:949-958.

27. Loewy AD, McKellar S: The neuroanatomical basis of central cardiovascular control. Fed Proc 1980, 39:2495-2503.

28. Brown RH Jr, Beyerl BD, Iseke R, Lavyne MH: Medulla oblongata edema associated with neurogenic pulmonary edema. Case report. J Neurosurg 1986, 64:494-500.

29. Imai K: Radiographical investigations of organic lesions of the hypothalamus in patients suffering from neurogenic pulmonary edema due to serious intracranial diseases: relationship between radiographical findings and outcome of patients suffering from neurogenic pulmonary edema. No Shinkei Geka 2003, 31:757-765.

30. Bahloul M, Chaari AN, Kallel H, et al: Neurogenic pulmonary edema due to traumatic brain injury: evidence of cardiac dysfunction. Am J Crit Care 2006, 15:462-470

31. Connor RC: Myocardial damage secondary to brain lesions. Am Heart J 1969, 78:145-148.

32. Mayer SA, Lin J, Homma S, et al:: Myocardial injury and left ventricular performance after subarachnoid hemorrhage. Stroke 1999, 30:780-786.

33. Zaroff JG, Rordorf GA, Ogilvy CS, Picard MH: Regional patterns of left ventricular systolic dysfunction after subarachnoid hemorrhage: evidence for neurally mediated cardiac injury. J Am Soc Echocardiogr 2000, 13:774-779.

34. Di Pasquale G, Andreoli A, Lusa AM, et al:: Cardiologic complications of subarachnoid hemorrhage. J Neurosurg Sci 1998, 42(Suppl 1):33-36.

35. Sarnoff SJ, Sarnoff LC: Neurohemodynamics of pulmonary edema. II. The role of sympathetic pathways in the elevation of pulmonary and stemic vascular pressures following the intracisternal injection of fibrin. Circulation 1952, 6:51-62

36. Minnear FL, Kite C, Hill LA, van der Zee H: Endothelial injury and pulmonary congestion characterize neurogenic pulmonary edema in rabbits. J Appl Physiol 1987, 63:335-341.

37. Carlson RW, Schaeffer RC Jr, Michaels SG, Weil MH: Pulmonary edema following intracranial hemorrhage. Chest 1979, 75:731-734.

38. van der Zee H, Malik AB, Lee BC, Hakim TS: Lung fluid and protein exchange during intracranial hypertension and role of sympathetic mechanisms. J Appl Physiol 1980, 48:273-280.

39. Theodore J, Robin ED: Speculations on neurogenic pulmonary edema (NPE). Am Rev Respir Dis 1976, 113:405-411.

40. Maron MB: Effect of elevated vascular pressure transients on protein permeability in the lung. J App/ Physiol 1989, 67:305-310.

41. Bosso FJ, Lang SA, Maron MB: Role of hemodynamics and vagus nerves in development of fibrin-induced pulmonary edema. J App/ Physio/ 1990 69:2227-2232

42. Wray NP, Nicotra MB: Pathogenesis of neurogenic pulmonary edema. Am Rev Respir Dis 1978, 118:783-786.

43. Richardson JB: Innervation of the pulmonary circulation: an overview. In The Pulmonary Circulation in Health and Disease. Edited by Will JA. Orlando: Academic Press: 1987:9-14.

44. McClellan MD, Dauber IM, Weil JV: Elevated intracranial pressure increases pulmonary vascular permeability to protein. J Appl Physiol 1989, 67:1185-1191

45. Peterson BT, Ross JC, Brigham KL: Effect of naloxone on the pulmonary vascular responses to graded levels of intracranial hypertension in anesthetized sheep. Am Rev Respir Dis 1983, 128:1024-1029.

46. Keegan MT, Lanier WL: Pulmonary edema after resection of a fourth 
ventricle tumor: possible evidence for a medulla-mediated mechanism. Mayo Clin Proc 1999, 74:264-268.

47. Fein A, Grossman RF, Jones JG, et al:: The value of edema fluid protein measurement in patients with pulmonary edema. Am J Med 1979, 67:32-38.

48. Fein IA, Rackow EC: Neurogenic pulmonary edema. Chest 1982, 81:318-320.

49. Wohns RN, Tamas L, Pierce KR, Howe JF: Chlorpromazine treatment for neurogenic pulmonary edema. Crit Care Med 1985, 13:210-211.

50. Malik AB: Mechanisms of neurogenic pulmonary edema. Circ Res 1985, $57: 1-18$.
51. Minnear FL, Connell RS: Prevention of aconitine-induced neurogenic pulmonary edema (NPE) with hypovolemia or methylprednisolone. J Trauma 1982, 22:121-128.

doi:10.1186/cc11226

Cite this article as: Davison DL, et al:: Neurogenic pulmonary edema. Critical Care 2012, 16:212. 